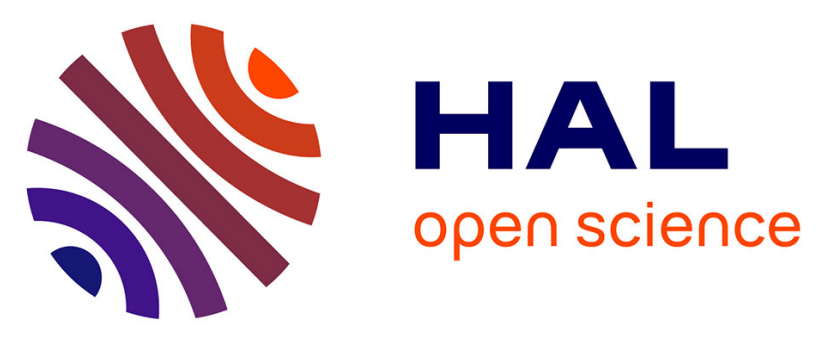

\title{
PDE2 regulates membrane potential, respiration and permeability transition of rodent subsarcolemmal cardiac mitochondria
}

Dawei Liu, Zhenyu Wang, Valérie Nicolas, Marta Lindner, Delphine Mika, Grégoire Vandecasteele, Rodolphe Fischmeister, Catherine Brenner

\section{To cite this version:}

Dawei Liu, Zhenyu Wang, Valérie Nicolas, Marta Lindner, Delphine Mika, et al.. PDE2 regulates membrane potential, respiration and permeability transition of rodent subsarcolemmal cardiac mitochondria. Mitochondrion, 2019, 47, pp.64-75. 10.1016/j.mito.2019.05.002 . hal-02356190

\author{
HAL Id: hal-02356190 \\ https://hal.science/hal-02356190
}

Submitted on 22 Oct 2021

HAL is a multi-disciplinary open access archive for the deposit and dissemination of scientific research documents, whether they are published or not. The documents may come from teaching and research institutions in France or abroad, or from public or private research centers.
L'archive ouverte pluridisciplinaire $\mathbf{H A L}$, est destinée au dépôt et à la diffusion de documents scientifiques de niveau recherche, publiés ou non, émanant des établissements d'enseignement et de recherche français ou étrangers, des laboratoires publics ou privés.

\section{(ㅇ)(1) $\$$}

Distributed under a Creative Commons Attribution - NonCommerciall 4.0 International 


\title{
PDE2 regulates membrane potential, respiration and permeability transition of rodent subsarcolemmal cardiac mitochondria
}

\author{
LIU Dawei $^{1 *}$, WANG Zhenyu ${ }^{1 *}$, NICOLAS Valérie ${ }^{2}$, LINDNER Marta $^{1}$, MIKA Delphine ${ }^{1}$, \\ VANDECASTEELE Grégoire ${ }^{1}$, FISCHMEISTER Rodolphe ${ }^{1}$, and BRENNER Catherine ${ }^{1}$
}

${ }^{1}$ INSERM UMR-S 1180, Faculty of Pharmacy, Univ Paris-Sud, Université Paris-Saclay, Châtenay-Malabry, France

${ }^{2}$ IPSIT-US31-UMS3679, Faculty of Pharmacy, Univ Paris-Sud, Université Paris-Saclay, Châtenay-Malabry, France

*: contributed equally to the work

Short title: cardiac mitochondrial PDEs

Correspondance to Dr Catherine Brenner

INSERM UMR-S 1180

Faculté de Pharmacie

Université Paris-Sud

5 Rue J.-B. Clément

92296 Châtenay-Malabry Cedex

France.

E-mail: catherine.brenner@u-psud.fr

\begin{abstract}
Abbreviations: $\Delta \Psi \mathrm{m}$, mitochondrial membrane potential; Bay, Bay 60-7550; Cil, Cilostamide; CsA, cyclosporine A; FCCP, Carbonyl cyanide-ptrifluoromethoxyphenylhydrazone; cilostamide, $\mathrm{Cil}$; $\mathrm{HCO}_{3}^{-}$, bicarbonate; $\mathrm{HF}$, heart failure; IFM, interfibrillar mitochondria, IM, inner membrane; OXPHOS, oxidative phosphorylation, sAC, soluble adenylyl cyclase; SSM, subsarcolemmal mitochondria, PDE, phosphodiesterase; Ro, Ro 20-1724; VDAC, voltage-dependent anion channel
\end{abstract}




\begin{abstract}
Cyclic adenosine monophosphate (cAMP) production regulates certain aspects of mitochondria function in rodent cardiomyocytes, such as ATP production, oxygen consumption, calcium import and mitochondrial permeability transition (MPT), but how this cAMP pool is controlled is not well known. Here, expression, localization and activity of several cAMP-degrading enzymes, i.e. phosphodiesterases (PDEs), were investigated in isolated rodent cardiac mitochondria. In contrast to the heart ventricle where PDE4 is the major PDE, in cardiac mitochondria, cGMP-stimulated PDE2 activity was largest than PDE3 and PDE4 activities. PDE2 expression was mainly detected in subsarcolemmal mitochondria in association with the inner membrane rather than in interfibrillar mitochondria. PDE2, 3 and 4 activities were further confirmed in neonatal rat cardiomyocytes by real time FRET analysis. In addition, the pharmacological inhibition or the cardiac-specific overexpression of PDE2 modulated mitochondrial membrane potential loss, MPT and calcium import. In mitochondria isolated from PDE2 transgenic mice with a cardiac selective PDE2 overexpression, the oxidative phosphorylation (OXPHOS) was significantly lower than in wild-type mice, but stimulated by cGMP. Thus, cAMP degradation by PDEs represents a new regulatory mechanism of mitochondrial function.
\end{abstract}

Keywords: calcium, cAMP, mitochondrial membrane potential, PDE, respiration 


\section{Introduction}

The heart is a highly energy-dependent organ with most of its energy provided by mitochondrial oxidative phosphorylation (OXPHOS). Mitochondria also play a role in many other essential cellular processes including metabolite synthesis, redox balance, calcium $\left(\mathrm{Ca}^{2+}\right)$ homeostasis and cell death, notably trough a sustained mitochondrio-nuclear dialog 1,2, 3. Therefore, maintaining a functional population of mitochondria is critical for cardiac function and identification of novel regulatory mechanisms of mitochondrial function is a crucial challenge. Recently, a soluble adenylyl cyclase (sAC) stimulated by bicarbonate $\left(\mathrm{HCO}_{3}{ }^{-}\right)$and calcium $\left(\mathrm{Ca}^{2+}\right)$ has been revealed to serve as a local source of the second messenger cAMP in the mitochondrial matrix. In liver and brain, mitochondrial cAMP stimulates ATP production by OXPHOS in response to $\mathrm{HCO}_{3}{ }^{-}$and/or $\mathrm{Ca}^{2+4,5}$. In endothelial cells, $\mathrm{HCO}_{3}^{-}$is transported into mitochondria leading to cAMP production by sAC, which indirectly modulates the cell fate trough apoptosis inhibition ${ }^{67}$. As a result, this pathway serves as a mechanism for metabolic adaptation and could be a potential novel target to treat genetic mitochondrial diseases ${ }^{4}$. In line with these studies, we recently showed a protective role for cAMP against cell death, apoptosis as well as necrosis in primary rat neonatal cardiomyocytes. Moreover, upon stimulation with $\mathrm{HCO}_{3}{ }^{-}$and $\mathrm{Ca}^{2+}$, a truncated form of $\mathrm{sAC}$ $(\mathrm{sACt})$ localized in mitochondria produces cAMP, which in turn stimulates oxygen $\left(\mathrm{O}_{2}\right)$ consumption, increases the mitochondrial membrane potential $(\Delta \Psi \mathrm{m})$ and ATP production. In addition, cAMP is rate-limiting for matrix $\mathrm{Ca}^{2+}$ entry via the exchange factor Epac1 and the mitochondrial $\mathrm{Ca}^{2+}$ uniporter and, as a consequence, prevents $\mathrm{MPT}^{8}$.

Cyclic nucleotide phosphodiesterases (PDEs) are a class of key cellular enzymes, which degrade cAMP and/or cGMP by hydrolysis of phosphodiester bonds ${ }^{9}$. Thereby, they regulate intracellular levels of these ubiquitous second messengers. Cyclic nucleotides play an important role in the regulation of a host of cellular targets involved in cardiac function but 
also in the progression of cardiac diseases such as heart failure ${ }^{10}$. In mammals, PDEs constitute a large superfamily which contains 11 PDE families (PDE1 to PDE11) and nongeneric PDEs such as the protein human Prune, PDE2 and PDE3 being respectively activated and inhibited by cGMP 11,12,13,14.

In isolated mitoplasts from mouse liver, cAMP catabolic activity is fully inhibited by 3isobutyl-1-methylxanthine (IBMX, a non-selective PDE inhibitor), suggesting the presence of intra-mitochondrial PDE activity ${ }^{4}$. It has been reported that PDE4B co-localized with mitochondria, apparently through an interaction with the disrupted in schizophrenia 1 (DISC1) protein ${ }^{15}$. More recently, mitochondrial PDE2A was shown to be located in the mitochondrial matrix and the unique $\mathrm{N}$ terminus of PDE2A isoform 2 was shown to be responsible for mitochondrial localization of this isoform in liver and brain ${ }^{5,16}$. Thus, mitochondrial PDE2 may form a local signalling system with sAC in the matrix, to regulate the activity of the respiratory chain ${ }^{5}$. PDE2 can also be found at the outer and inner mitochondrial membranes regulating mitochondrial morphology and apoptotic cell death via local modulation of a cAMP/PKA signalling module facing the cytosol ${ }^{17}$. In Drosophila, the cyclic nucleotide PDE Prune was also found to localize to the mitochondrial matrix. Knocking-down Prune reduces mitochondrial transcription factor A (TFAM) and mitochondrial DNA levels, suggesting that Prune stabilizes TFAM and promotes mitochondrial DNA replication through down-regulation of mitochondrial cAMP signalling 18.

To gain new insights into the control and degradation of the cardiac mitochondrial pool of cAMP, we assessed the presence and contribution of various isoforms of phosphodiesterases (PDEs) to cAMP degradation in isolated rat or mouse heart mitochondria in the presence and absence of cGMP. Our results show that PDE2A, 3A, 4A, 4B and 4D can be detected within mitochondria. These PDEs control cAMP level to regulate $\mathrm{Ca}^{2+}$ accumulation and certain 
mitochondrial functions. Our findings unravel the role of cAMP degradation by mitochondrial PDEs, which represents a new regulatory mechanism of mitochondrial function.

\section{Materials and Methods}

Unless specified, all reagents and chemicals are from SIGMA Aldrich and of analytical grade.

\subsection{Animals}

All animal care and experimental procedures conformed to the European Community guiding principles in the care and use of animals (Directive 2010/63/EU of the European Parliament) and authorizations to perform animal experiments according to this decree were obtained from the French Ministry of Agriculture, Fisheries and Food (No. D-92-283, 13 December 2012). All studies involving rats are reported in accordance with the ARRIVE guidelines for reporting experiments involving animals ${ }^{19}$.

\subsection{Isolation of rat and mouse cardiac mitochondria}

Subsarcolemmal mitochondria (SM) were isolated from the heart of adult Wistar rats at 8-10 weeks of age (275-375 g; Janvier, Le Genest St Isle, France) and from mice heart at 15-20 weeks of age (Janvier, Le Genest St Isle, France) as described ${ }^{8}$. Briefly, over-night fasted rats were decapitated and mouse killed by cervival elongation in accordance with the rules of our animal facility. The heart and its atria were rapidly removed, rinsed to discard blood traces, and weighted. Then, ventricles were placed into a cold homogenization buffer containing 0.3 M sucrose, $0.2 \mathrm{mM}$ EGTA, $5 \mathrm{mM}$ TES (pH 7.2). The heart was grinded with Polytron fastly and homogenized by using a Potter on ice. The homogenate was centrifuged at $500 \mathrm{~g}$ for 10 $\min , 4^{\circ} \mathrm{C}$. Then the supernatant was carefully removed and centrifuged again at $12,000 \mathrm{~g}$ for $15 \mathrm{~min}, 4^{\circ} \mathrm{C}$. The pellets were washed in the homogenization buffer supplemented with $1 \mathrm{~g} / \mathrm{L}$ bovine serum albumin (BSA), called buffer $\mathrm{H}-\mathrm{BSA}_{2}$ and mitochondria were kept on ice until use. To isolate interfibrillar mitochondria, the proteolytic enzyme trypsin ( $5 \mathrm{mg} / \mathrm{g}$ of tissue) 
was added to the homogenate fraction and immediately centrifuged at 12,000 $\mathrm{g}$ for $15 \mathrm{~min}$ at $4^{\circ} \mathrm{C}$. Then, the pellet was suspended in buffer $\mathrm{H}$-BSA and centrifuged at $1,000 \mathrm{~g}$ for $10 \mathrm{~min}$ at $4^{\circ} \mathrm{C}$. The supernatant was centrifuged at $12,000 \mathrm{~g}$ for $15 \mathrm{~min}, 4^{\circ} \mathrm{C}$ and mitochondria were kept on ice until use.

\subsection{Mitoplast preparation}

Freshly isolated mitochondria were incubated in $\mathrm{H}_{2} \mathrm{O}$ containing $15 \mathrm{mM} \mathrm{KCl}$ for $30 \mathrm{~min}$ at $4^{\circ} \mathrm{C}$ to induce an osmotic shock and then collected by centrifugation at 3,000 $\mathrm{g}$ for $10 \mathrm{~min}$, $4^{\circ} \mathrm{C}$

\subsection{Measurement of mitochondrial transmembrane potential and swelling in isolated mitochondria}

Isolated mitochondria (25 $\mu \mathrm{g}$ proteins) were incubated with $\mathrm{Ca}^{2+}$ and drugs in 96 well microtiter plates ${ }^{8}$. Mitochondrial transmembrane potential was measured using the fluorescent probe, rhodamine $123($ Rhod123, Excitation $=485 \mathrm{~nm}$ and Emission $=535 \mathrm{~nm}$, Enzo Life Sciences, Villeurbanne, France) in a buffer containing $200 \mathrm{mM}$ sucrose, $10 \mathrm{mM}$ MOPS, $200 \mu \mathrm{M}$ EGTA, $1 \mathrm{mM} \mathrm{H} \mathrm{H}_{3} \mathrm{PO}_{4}, 5 \mathrm{mM}$ succinate and $2 \mu \mathrm{M}$ rotenone (pH 7.4) using Tecan Infinite 200 spectrofluorimeter. In parallel, matrix swelling was measured via absorbance at $540 \mathrm{~nm}^{8}$. Raw results were exported to an Origin8 (OriginLab Corporation) files and half-time of effects were calculated using nonlinear curve fitting with a logistic fit.

\subsection{Measurement of mitochondrial $\mathrm{Ca}^{2+}$ in isolated mitochondria}

Isolated mitochondria (25 $\mu \mathrm{g}$ proteins) were incubated with $5 \mu \mathrm{M}$ Rhod-2 (Enzo Life Sciences, Villeurbanne, France) in the buffer containing $200 \mathrm{mM}$ sucrose, $10 \mathrm{mM}$ MOPS, 200 $\mu \mathrm{M}$ EGTA, $1 \mathrm{mM} \mathrm{H}_{3} \mathrm{PO}_{4}, 5 \mathrm{mM}$ succinate and $2 \mu \mathrm{M}$ rotenone for $30 \mathrm{~min}$ in dark at room temperature. The mitochondria were then washed 2 times and were treated with various drugs for $10 \mathrm{~min}$ before applying $\mathrm{Ca}^{2+}$. Fluorescence was measured in real time for $60 \mathrm{~min}$ at room 
temperature (RT) in 96-well plates using Tecan Infinite 200 (excitation at $552 \mathrm{~nm}$; Emission at $581 \mathrm{~nm})$.

\section{6 cAMP measurements by ELISA}

Cyclic AMP measurement was performed according to the manufacturer's instructions using monoclonal anti-cAMP antibody based direct cAMP ELISA Kit (R\&D, Lille, France) on freshly isolated mitochondria from rat hearts $(500 \mu \mathrm{g}$ proteins per sample) treated or not by $\mathrm{HCO}_{3}{ }^{-}, \mathrm{PDE}$ inhibitors and $5 \mu \mathrm{M}$ cGMP for $20 \mathrm{~min}$ at RT before centrifugation and lysis ${ }^{8}$.

\subsection{Cyclic AMP-PDE activity assay}

The cAMP-PDE activity was measured in the supernatant according to a modification of the two-step assay procedure method described by Thompson and Appleman ${ }^{20}$, using $20 \mu \mathrm{g}$ of heart homogenate proteins / $200 \mu \mathrm{g}$ of mitochondria proteins in a total volume of $200 \mu \mathrm{l}$ of

buffer A, which includes $10 \mathrm{mM}$ Tris-HCl, $\mathrm{pH} 8.0,10 \mathrm{mM} \mathrm{MgCl} 2,5 \mathrm{mM} \beta$-mercaptoethanol and $1 \mu \mathrm{M}$ cAMP and supplemented with $10^{5} \mathrm{cpm}\left[{ }^{3} \mathrm{H}\right]-\mathrm{cAMP}$, as detailed previously ${ }^{21}$. To evaluate PDE family-specific activities, the assay was performed in the absence or presence of either one or a combination of several selective PDE inhibitors: $100 \mathrm{nM}$ Bay 60-7550 (Bay) for PDE2, $1 \mu \mathrm{M}$ Cilostamide (Cil) for PDE3, $10 \mu \mathrm{M}$ Ro 20-1724 (Ro) for PDE4 and $500 \mu \mathrm{M}$ IBMX as a non-selective PDE inhibitor. In some experiments, $5 \mu \mathrm{M}$ cGMP was added to the buffer A to activate PDE2 and inhibit PDE3.

\subsection{Oxygen consumption analysis in isolated mitochondria}

The XF 96 Extracellular Flux Analyzer (Seahorse Biosciences, North Billerica, MA, USA) was used to measure mitochondrial bioenergetic function ${ }^{22}$. Respiration by the mitochondria $(0.5 \mu \mathrm{g} /$ well) was sequentially measured in a coupled state with substrate (basal respiration, state 2). State 3 initiated with $2 \mathrm{mM}$ ADP, state 4 induced with the addition of $2.5 \mu \mathrm{g} / \mathrm{ml}$ oligomycin and $2 \mu \mathrm{M}$ FCCP-induced maximal uncoupler-stimulated respiration (state $3 \mathrm{u}$ ) 
were sequentially measured. To activate PDE2 and inhibit PDE3, $5 \mu \mathrm{M}$ cGMP was added at the beginning of the experiment to the isolated mitochondria during $20 \mathrm{~min}$ at RT. At the end of the experiment, the complex III inhibitor, antimycin A was applied at $4 \mu \mathrm{M}$ to completely inhibit the mitochondrial respiration. This 'coupling assay' examines the degree of coupling between the electron transport chain (ETC), and OXPHOS.

\subsection{SDS PAGE and Western blotting}

Total heart homogenate and total mitochondrial proteins were resolved on 4-15\% Tris-glycine SDS-PAGE gels and electroblotted onto polyvinylidene fluoride (PVDF) membranes (BioRad, Marnes La Coquette, France). Following electrotransfer, membranes were blocked for 1 $\mathrm{h}$ at room temperature in 5\% BSA-PBST (10 mM Tris $\mathrm{HCl}, \mathrm{pH} 8.0 / 150 \mathrm{mM} \mathrm{NaCl} / 0.1 \%$ Tween 20). Next, membranes were incubated overnight at $4{ }^{\circ} \mathrm{C}$ with primary antibody. Then, the membranes were washed six times with PBST and incubated with peroxidase-conjugated secondary antibody at room temperature for $1 \mathrm{~h}$. Peroxidase activity was detected with enhanced chemiluminescence (ECL Advance Western blotting detection kit; Thermo Scientific, Villebon sur Yvette, France). For protein detection, the following antibodies were used: anti PDE2A (G-12, Santa Cruz, CA, USA), anti PDE3A (generous gift from Pr. Chen Yan, University of Rochester, USA), anti PDE4A, PDE4B and PDE4D (generous gifts from Pr. Marco Conti, University of California, San Francisco, USA), anti GAPDH (Cell signalling, Danvers, MA, USA) and anti voltage-dependent anion channel (VDAC) (Genosphere, Paris, France).

\subsection{Neonatal cardiomyocyte isolation and adenoviral infection}

Rat neonatal cardiomyocytes were isolated as previously described ${ }^{23}$. For FRET experiments, neonatal cardiomyocytes were plated on $35 \mathrm{~mm}$ laminin-coated culture dishes $(10 \mu \mathrm{g} / \mathrm{ml})$ at a density of $4 \times 10^{5}$ cells per dish. On the following day, cells were infected with an adenovirus encoding the cAMP fluorescent reporter $4 \mathrm{mt}-E p a c-S^{\mathrm{H} 187}$ in Opti-MEM® (Life technologies, 
St Aubin, France) for 48h with 100 of MOI (multiplicity of infection) . The construction of adenoviruse encoding $4 \mathrm{mt}-$ Epac-SH187 has been described previously ${ }^{8}$.

\subsection{Fluorescence resonance energy transfer measurements of cAMP levels}

Fluorescence resonance energy transfer (FRET) imaging experiments were performed $48 \mathrm{~h}$ after infection of rat neonatal cardiomyocytes with the $4 \mathrm{mt}-\mathrm{Epac}-\mathrm{S}^{\mathrm{H} 187}$ adenovirus. Cells were bathed in Hepes-buffered Ringer's solution containing: $125 \mathrm{mM} \mathrm{NaCl}, 25 \mathrm{mM}$ Hepes, $10 \mathrm{mM}$ glucose, $5 \mathrm{mM} \mathrm{K}_{2} \mathrm{HPO}_{4}, 1 \mathrm{mM} \mathrm{MgSO}$, and $1 \mathrm{mM} \mathrm{CaCl}_{2}$, pH 7.4. For sAC activation by $\mathrm{HCO}_{3}{ }^{-}$, the medium was the Krebs-Henseleit solution containing: $120 \mathrm{mM} \mathrm{NaCl}, 2.09 \mathrm{mM}$ $\mathrm{K}_{2} \mathrm{HPO}_{4}, 0.34 \mathrm{mM} \mathrm{KH} \mathrm{PO}_{4}, 24 \mathrm{mM} \mathrm{NaHCO} 3,1 \mathrm{mM} \mathrm{MgSO} 4,1 \mathrm{mM} \mathrm{CaCl}_{2}$, and $10 \mathrm{mM}$ Dglucose. Krebs-Henseleit solution was gassed continuously with $95 \% \mathrm{O}_{2} / 5 \% \mathrm{CO}_{2}$ to maintain a $\mathrm{pH}$ of 7.4. Real-time FRET experiments were performed at room temperature. Images were captured every $5 \mathrm{~s}$ using the $40 \times$ oil immersion objective of an inverted microscope (Nikon, Champigny sur Marne, France) connected to a Cool SNAP HQ2 camera (Photometrics, Tucson, AZ, USA) controlled by the Metafluor software (Molecular Devices, Sunnyvale, CA, USA). The donor (mTurquoise 2$)^{24}$ was excited during $300 \mathrm{~ms}$ by a Xenon lamp (Nikon) using a 440/20BP filter and a 455LP dichroic mirror. Dual-emission imaging of donor and acceptor was performed using a Dual-View emission splitter equipped with a 510 LP dichroic mirror and BP filters 480/30 and 535/ $25 \mathrm{~nm}$, respectively.

\subsection{Immunostaining}

Cultured mouse cardiomyocytes were rinsed with PBS and fixed with $4 \%$ formaldehyde. Triton X-100-permeabilized cells were incubated with anti PDE2 antibody (Santa Cruz, CA, USA) or anti alpha actinin (A7811, Sigma). Cells were then incubated with, respectively, AlexaFluor goat anti-mouse secondary antibody (ThermoFisher scientific, Courtaboeuf, France) or AlexaFluor anti-rabbit secondary antibody (ThermoFisher scientific), and viewed 
under a fluorescent microscope in inverted confocal laser scanning microscope LSM 510Meta (Carl Zeiss, Germany) using a Plan-Apochromat 63X/1.4 objective lens, equipped with an argon (488 $\mathrm{nm}$ excitation wavelength) and a helium neon laser (543 $\mathrm{nm}$ excitation wavelength). Sections stained by immunofluorescence were visualized by confocal microscopy and intensity profile obtained. To calculate the Pearson correlation coefficient, we used the plugin JACop in Image J software ${ }^{25}$. The coefficient is equal to -1 when there is a negative relationship between two parameters, +1 when there is a positive relationship and 0 when there is no relationship.

\subsection{Electron microscopy}

Isolated mitochondria were fixed in $4 \%$ paraformaldehyde and $0.5 \%$ glutaraldehyde in $0.1 \mathrm{M}$ sodium phosphate buffer $\mathrm{pH} 7.4$ during $2 \mathrm{~h}$, followed by $4 \%$ paraformaldehyde for $3 \mathrm{~h}$. Samples were gradually dehydrated in ethanol (30-100\%) on ice and embedded in LRW resin, polymerised for $24 \mathrm{~h}$ at $60^{\circ} \mathrm{C}$. Thin sections $(80 \mathrm{~nm})$ were collected onto 300 mesh nickel grids, and processed for immunochemistry with anti-PDE2 antibodies. As a control, we checked that the gold nanoparticle conjugated antibody gives no background on the section of isolated mitochondria. The observation was processed with a Philips CM12 transmission electron microscope at $80 \mathrm{kV}$.

\subsection{Statistical data analysis}

Results are expressed as mean \pm standard error of mean (s.e.m). The Origin software and Graphpad Prism 6 were used for statistical analysis. Differences between 2 groups have been analyzed by one-way ANOVA and differences between groups of two genotypes were analysed by two way ANOVA, Sidak's multiple comparisons. Statistical significance is indicated as following: $* P<0.05$, $* * P<0.01$, $* * * P<0.001$, $* * * * P<0.0001$. The number of animals, cells and independent experiments performed is indicated in the figure legends. 


\section{Results}

3.1 PDE2, PDE3 and PDE4 isoforms are expressed in cardiac mitochondria To identify PDE expression pattern in cardiac mitochondria, we isolated mitochondria from rat heart ventricles by differential centrifugation and extensive washes to minimize cross contamination by subcellular fractions ${ }^{8}$. The analysis of rat heart homogenate $(\mathrm{H})$ and of isolated mitochondria $(\mathrm{M})$ revealed several bands at apparent molecular weights comprised between $95 \mathrm{kDa}$ and $130 \mathrm{kDa}$-for PDE2A, PDE3A, PDE4A, PDE4B and PDE4D subtypes in western-blots (Fig. 1A). Because PDE2A was reported to be localized in the mitochondrial matrix in liver and brain ${ }^{416}$, whereas it was proposed to be localized mainly at the OMM and IMM in neonatal rat cardiomyocytes ${ }^{17}$, we next focused on PDE2 and explored its localization in mitochondrial subcompartments using fractionation followed by osmotic shock to prepare mitoplasts. Fig. 1B shows that a PDE2 band was detected in the mitoplast fraction but not in the post-mitoplast fraction, which corresponds to the intermembrane space and the outer membrane (Fig. 1B). To confirm this, we analyzed the mitochondrial population by transmission electron microscopy and immunolabelling. As shown in Fig. 1, we observed that not all mitochondria are labelled and found that the total mitochondria population (labelled+unlabelled, subsarcolemmal mitochondria $(\mathrm{SSM})+$ interfibrillar mitochondria (IFM) exhibits a mean of 0.8 dots/mitochondrion (Fig 1D). In labelled mitochondria, PDE2 was mainly found to be localized in the inner membrane and the matrix (Fig. 1C-D). Interestingly, PDE2 was 2-fold more expressed in SSM than in IFM (Fig. 1D), and 3-fold more present in the inner membrane than in the matrix (Fig. 1E). PDE2 was barely detected at the outer membrane, suggesting a low level of association with this membrane or the inaccessibility of epitopes due to PDE2 conformation or interaction with other proteins (Fig. 1E). In the following experiments, we used exclusively SSM mitochondria to investigate the role of PDEs. 
A
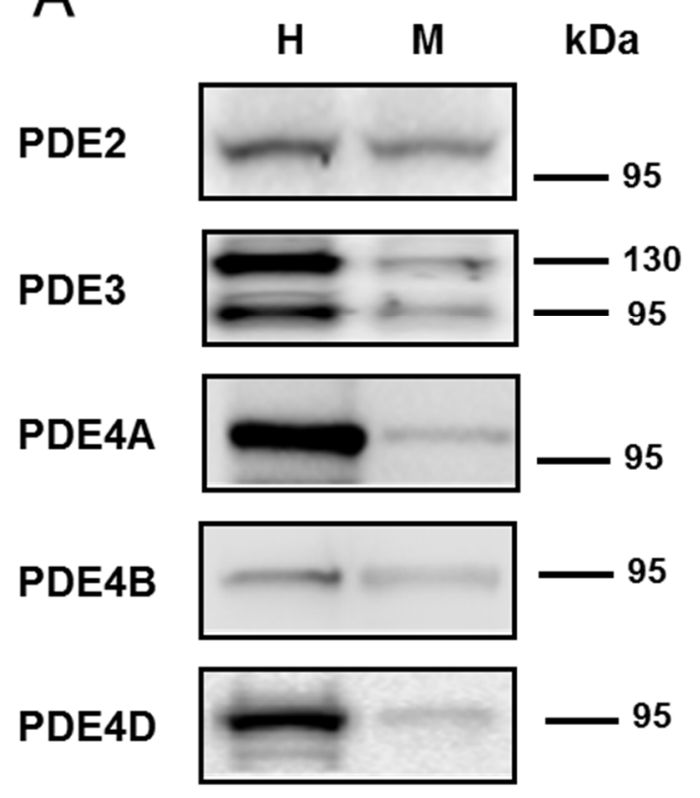

B

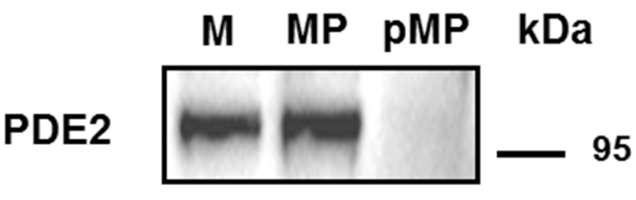

C subsarcolemmal mitochondria (SSM)

D

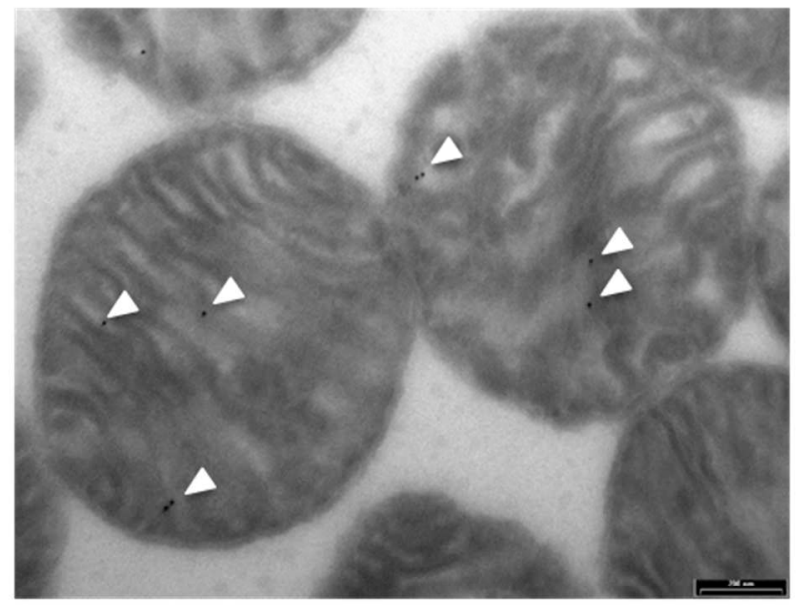

Interfibrillar mitochondria (IFM)

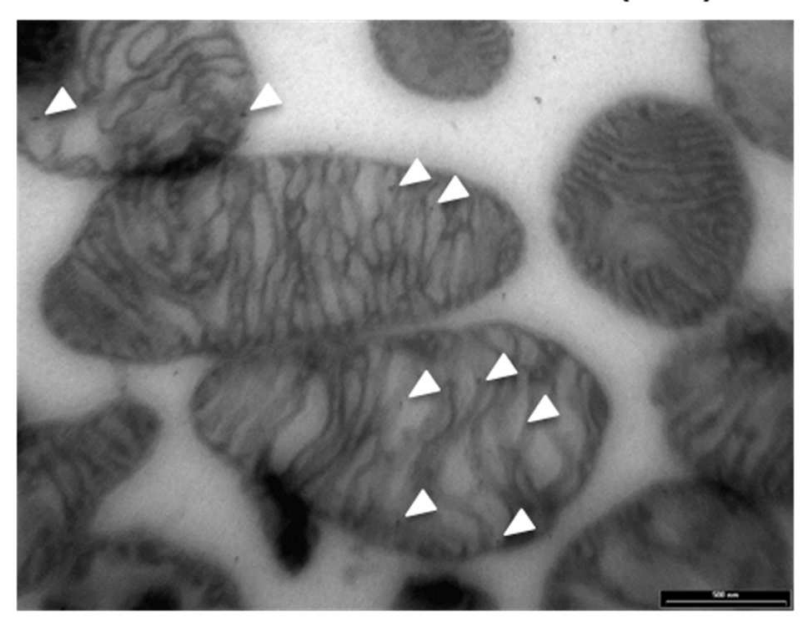

E

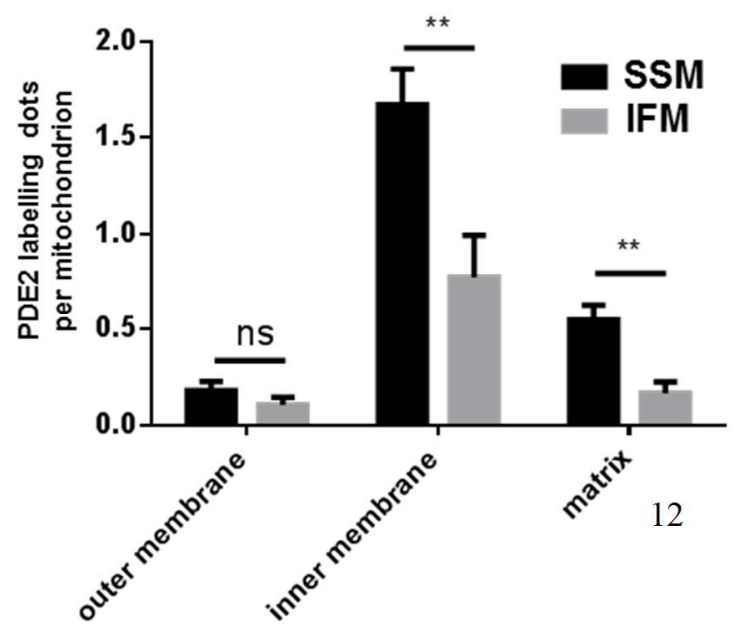

Fig. 1 
Figure 1. Expression of PDE isoforms in rat heart and mitochondria. (A) Expression of PDE2A, 3A, 4A, 4B and 4D in heart homogenate $(\mathrm{H})$ and total isolated mitochondria $(\mathrm{M})$ by western-blot. $30 \mu \mathrm{g}$ protein per lane. (B) PDE2A expression in mitochondria (M), mitoplast (MP) and post mitoplast (pMP). (C) Transmission electron microscopy analysis of PDE2A in subsarcolemmal mitochondria (SSM) and interfibrillar mitochondria (IFM) and quantification for total mitochondria (labelled+unlabeled) in (D) and for labelled mitochondria (E). At least 150 mitochondria were counted and the bargraphs show the mean \pm s.e.m. Statistical analysis was performed using t-test for (D) and one way ANOVA for (E). **, p< 0.01 and ns, nonsignificant. Scale bars in (C) represent $200 \mathrm{~nm}$ (top) and 500nm (bottom).

\section{2 cAMP-PDE activity in cardiac mitochondria}

To evaluate the cAMP-hydrolysing activity of the three different PDE families that we identified in SSM mitochondria, a radioenzymatic assay was conducted in the absence or presence of selective PDE inhibitors ${ }^{21}$ : Bay 60-7550 (Bay, $100 \mathrm{nM}$ ) for PDE2, cilostamide (Cil, $1 \mu \mathrm{M}$ ) for PDE3 and Ro 20-1724 (Ro, $10 \mu \mathrm{M}$ ) for PDE4, as well as in the absence or presence of $5 \mu \mathrm{M}$ cGMP to stimulate PDE2 and inhibit PDE3, respectively. As shown previously ${ }^{26}$, PDE4 was the major PDE in rat left ventricle (Fig. 2A \& 2B), representing $\sim 63 \%$ of total cAMP-hydrolyzing activity at $1 \mu \mathrm{M}$ cAMP and $\sim 74 \%$ in the additional presence of $5 \mu \mathrm{M}$ cGMP, this difference being due to decreased contribution of the cGMPinhibited PDE3 to the total activity (Fig. 2B, Table 1). In ventricle, PDE2 accounted for only $\sim 6 \%$ of total cAMP-hydrolyzing activity with cAMP alone, and increased to $12 \%$ in the 
presence of cGMP (Fig. 2B, Table 1). In contrast, in cardiac mitochondria, PDE2 activity represented about $22 \%$ of total cAMP-hydrolytic activity with cAMP alone and $63 \%$ in the presence of cGMP, indicating that in the presence of cGMP, PDE2 constituted the largest cAMP-PDE activity in cardiac mitochondria ${ }^{27}$ (Fig. 2C, D, Table 1). In line with the PDE activity assay, quantitative measurements of mitochondrial cAMP levels by ELISA showed that stimulation by $\mathrm{HCO}_{3}{ }^{-}$, a known activator of $\mathrm{sAC}$, increased the level of cAMP produced compared to untreated controls and that this increase was minored in the presence of cGMP (Fig. 2E). This result indicates that cGMP can directly affect cAMP catabolism in isolated mitochondria, most probably by activation of PDE2. Moreover, concomitant pharmacological inhibition of PDE2, 3 and 4 families by a combination of Bay, Cil and Ro also led to a significant increase in cAMP level. Finally, there was no impact of cGMP on $\mathrm{HCO}_{3}{ }^{-}$and the three PDEs inhibitors (Fig. 2E). 
A
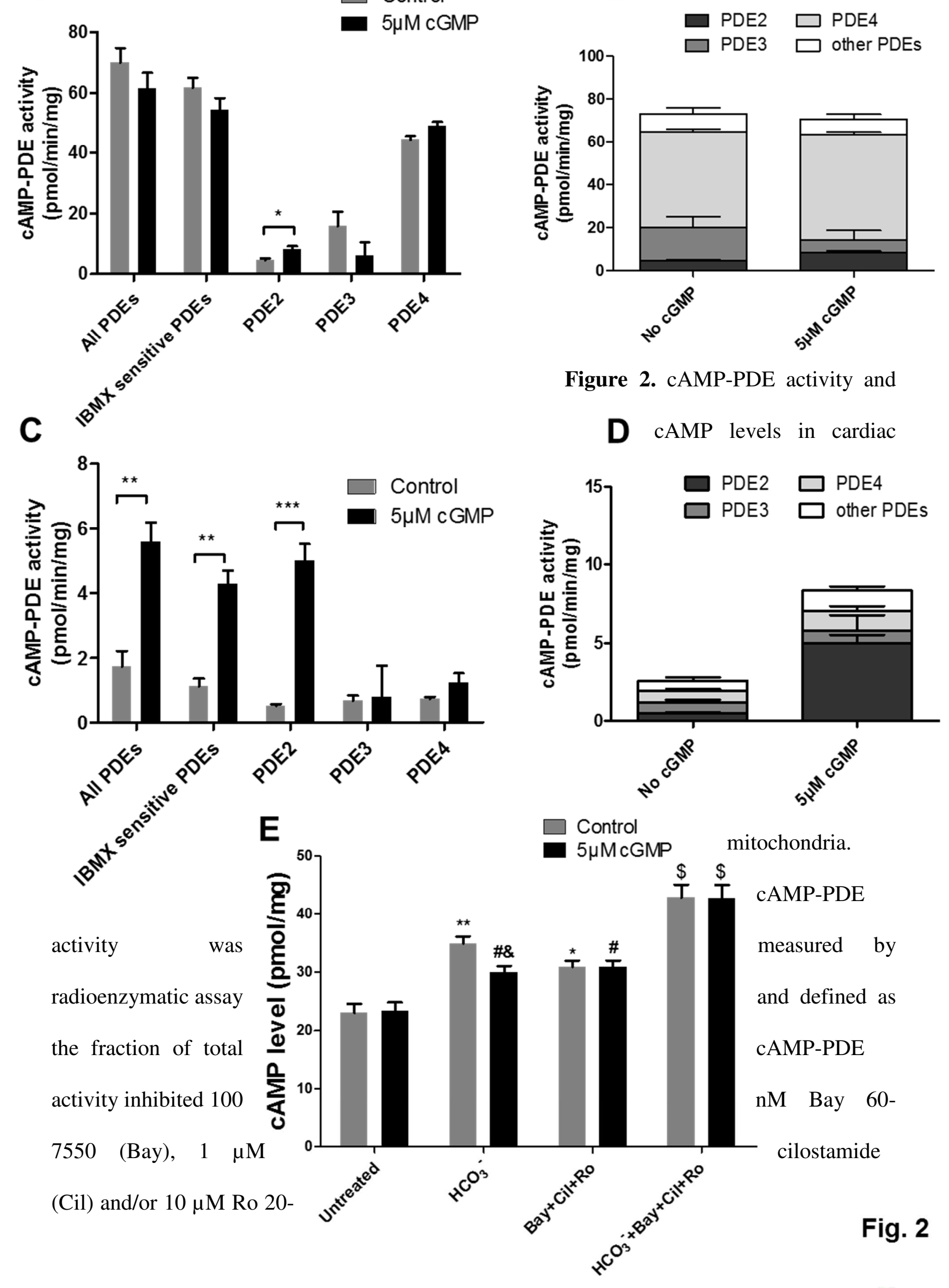

Figure 2. cAMP-PDE activity and

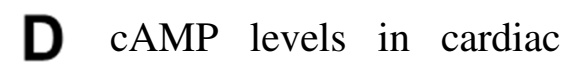


1724 (Ro) in heart ventricle homogenate (A, B) and SSM mitochondria (C, D) (n=3). (E) Mitochondrial cAMP levels were measured by ELISA under basal condition or following stimulation of soluble adenylyl cyclase with $\mathrm{HCO}^{-}(15 \mathrm{mM})+$ inhibition of PDE2 with Bay, PDE3 with Cil and PDE4 with Ro. The bar graphs show the mean \pm s.e.m of 4 individual experiments. Statistical analysis was performed using one way ANOVA. ${ }^{*} \mathrm{P}<0.05, * * \mathrm{P}<0.01$ vs untreated control group, $\# \mathrm{P}<0.05$ vs $5 \mu \mathrm{M}$ cGMP control group, \&P $<0.05$ vs $\mathrm{HCO}^{-}$ control group, $\$ \mathrm{P}<0.05$ vs $\mathrm{HCO}^{-}$group. 
Table 1. Relative proportion of PDE activities in ventricle homogenate vs mitochondria

\begin{tabular}{llcccc}
\hline & & $\begin{array}{c}\text { Other PDEs } \\
(\boldsymbol{\%})\end{array}$ & $\begin{array}{c}\text { PDE2 } \\
(\boldsymbol{\%})\end{array}$ & PDE3 (\%) & PDE4 (\%) \\
\hline $\begin{array}{l}\text { Heart } \\
\text { Ventricle }\end{array}$ & No cGMP & 9 & 6 & 22 & 63 \\
\cline { 2 - 6 } Homogenate & $5 \mu \mathrm{M}$ & 7 & 12 & 7 & 74 \\
\hline Mitochondria & No cGMP & 19 & 22 & 28 & 31 \\
\cline { 2 - 6 } & $\begin{array}{l}5 \mu \mathrm{M} \\
\text { cGMP }\end{array}$ & 14 & 63 & 9 & 14 \\
\hline
\end{tabular}




\subsection{FRET measurements of cAMP signals in cardiac mitochondria}

To determine whether the different PDEs regulate mitochondrial cAMP in primary cardiomyocytes, neonatal rat cardiomyocytes were transduced with an adenovirus encoding a cAMP sensitive FRET sensor (Epac-S $\mathrm{S}^{\mathrm{H} 18724}$ ) fused with a $4 \mathrm{mt}$ sequence. The localization of $4 \mathrm{mt}-$ Epac- $\mathrm{S}^{\mathrm{H} 187}$ in mitochondria was shown by co-localization of its green fluorescence with MitoTracker red fluorescence ${ }^{8}$. In basal condition, inhibition of PDE2 with Bay (100 nM, Fig 3A) and PDE3 with Cil (1 $\mu \mathrm{M}$, Fig 3B), respectively, caused a slight increase in mitochondrial cAMP level (2.5\% increase in CFP/YFP ratio). Inhibition of PDE4 with Ro (10 $\mu \mathrm{M})$ resulted in a much stronger increase in mitochondrial cAMP (25\% increase in CFP/YFP ratio, Fig. 3C). We next evaluated the effect of the different PDE families on cAMP degradation following sAC stimulation with $\mathrm{HCO}_{3}{ }^{-} \cdot \mathrm{HCO}_{3}{ }^{-}$alone caused a $15 \%$ increase in CFP/YFP ratio and a subsequent application of Bay or Cil resulted in a further increase in mitochondrial cAMP (Fig. Fig. 3D and E). Inhibition of PDE4 had larger effect on mitochondrial cAMP levels than inhibition of PDE2 or PDE3 (Fig. 3F). 

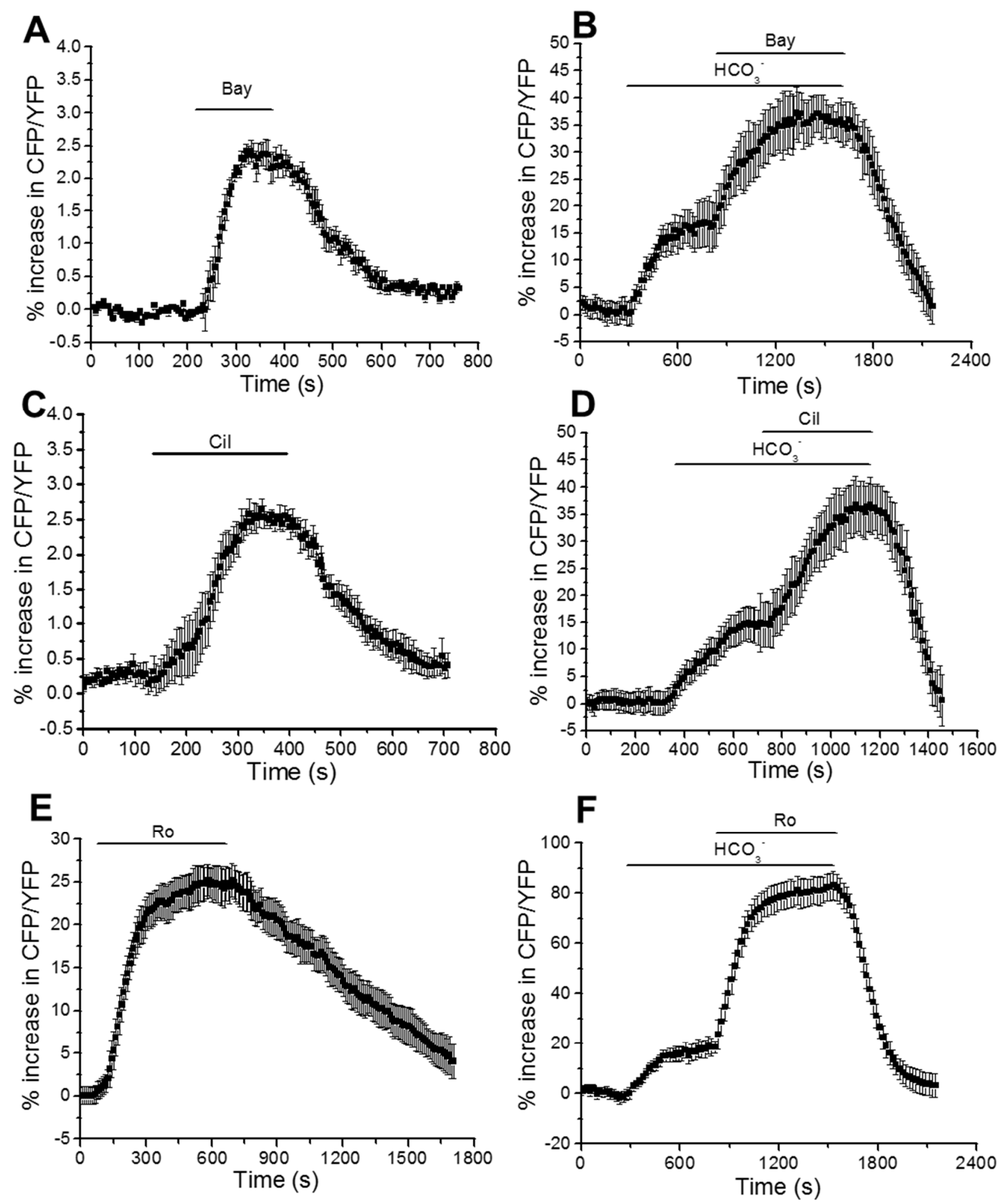

Fig. 3 
Figure 3. Effect of PDE inhibition on mitochondrial cAMP in the absence or presence of $\mathrm{HCO}_{3}{ }^{-}$in neonatal cardiomyocytes using a FRET-based cAMP probe. Time course of the CFP/YFP ratio recorded in rat neonatal cardiomyocytes infected with $4 \mathrm{mt}-$ Epac-S $\mathrm{S}^{\mathrm{H} 187}$ exposed to $100 \mathrm{nM}$ Bay (A, B), $1 \mu \mathrm{M}$ Cil (C, D) and $10 \mu \mathrm{M}$ Ro (E, F) in basal conditions (AC) or following application of $15 \mathrm{mM} \mathrm{HCO}_{3}^{-}(\mathbf{B}, \mathbf{D}, \mathbf{F})$. The data were obtained from three different isolations and each data point shows the mean \pm s.e.m of 10-15 cells.

\subsection{PDEs regulate $\mathrm{Ca}^{2+}$-induced mitochondrial permeability transition (MPT)}

MPT is an irreversible process of membrane permeabilisation that is characterized by a sudden matrix swelling and a loss of mitochondrial membrane potential $(\Delta \Psi \mathrm{m})$ in response to $\mathrm{Ca}^{2+}$ overload. MPT is thus an hallmark of dysfunctional mitochondria, since $\Delta \Psi \mathrm{m}$ is critical for allowing the respiratory chain activity to generate ATP ${ }^{28}$. Using spectrofluorimetry to measure $\Delta \Psi \mathrm{m}$ and matrix swelling, we and others previously showed that MPT can be elicited by $10 \mu \mathrm{M} \mathrm{Ca}^{2+}$ and prevented by $5 \mu \mathrm{M}$ cyclosporin A (CsA) in cardiac isolated mitochondria ${ }^{8}$. We also showed previously that cAMP elevation protects mitochondria from $\mathrm{Ca}^{2+}$-induced MPT ${ }^{8}$. We now examined whether PDEs could modulate the $\mathrm{Ca}^{2+}$-induced MPT response. For that, we used a robust miniaturized assay ${ }^{82}$ to concomitantly measure $\Delta \Psi \mathrm{m}$ level and swelling induced by $\mathrm{Ca}^{2+}$ as a function of time in the presence or absence of selective PDE inhibitors and cGMP. As shown in Fig. 4, PDE inhibition did not affect $\Delta \Psi \mathrm{m}$ loss or swelling induced by $\mathrm{Ca}^{2+}$ under basal condition. However, upon stimulation of soluble adenylyl cyclase with $\mathrm{HCO}_{3}$, both $\Delta \Psi \mathrm{m}$ loss and swelling were delayed. Inhibition of PDE2, 3 and 4 further slowed down both processes compared to $\mathrm{HCO}_{3}{ }^{-}$alone (Fig. 4). In our experimental conditions, both processes were not affected by $5 \mu \mathrm{M}$ cGMP pre-treatment in the presence of $\mathrm{Ca}^{2+}$ or not (not shown). 

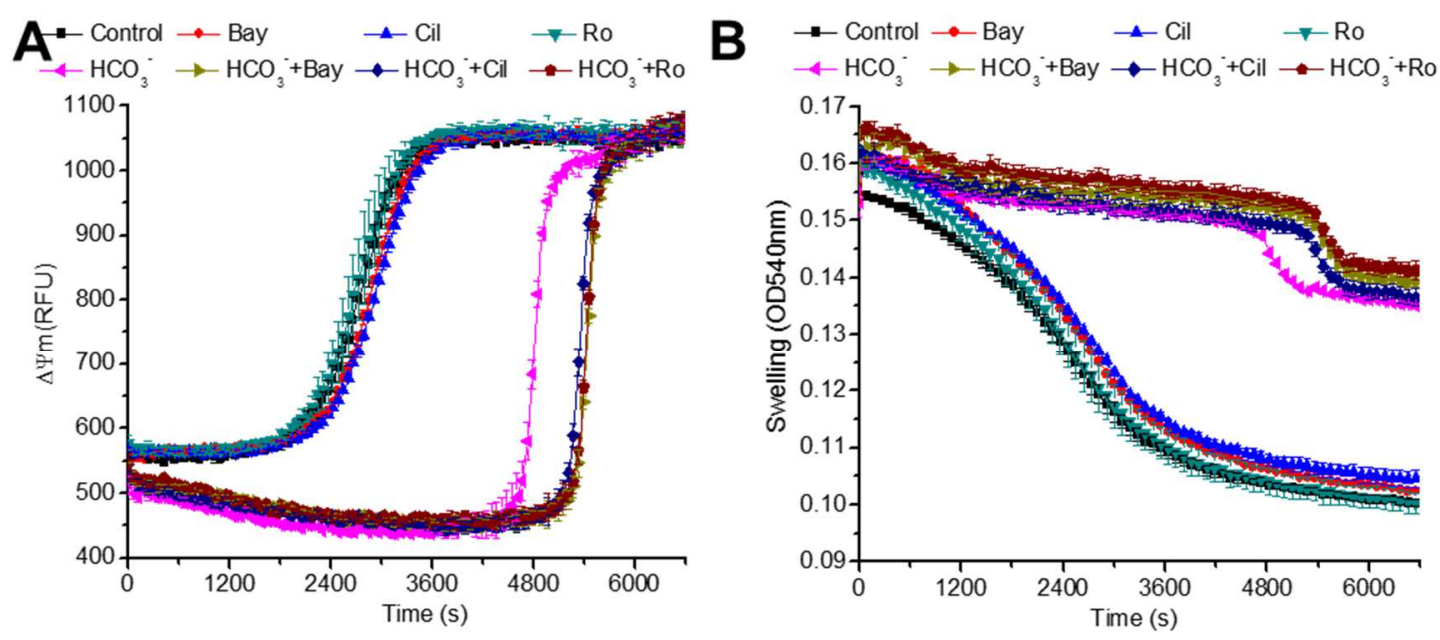

C

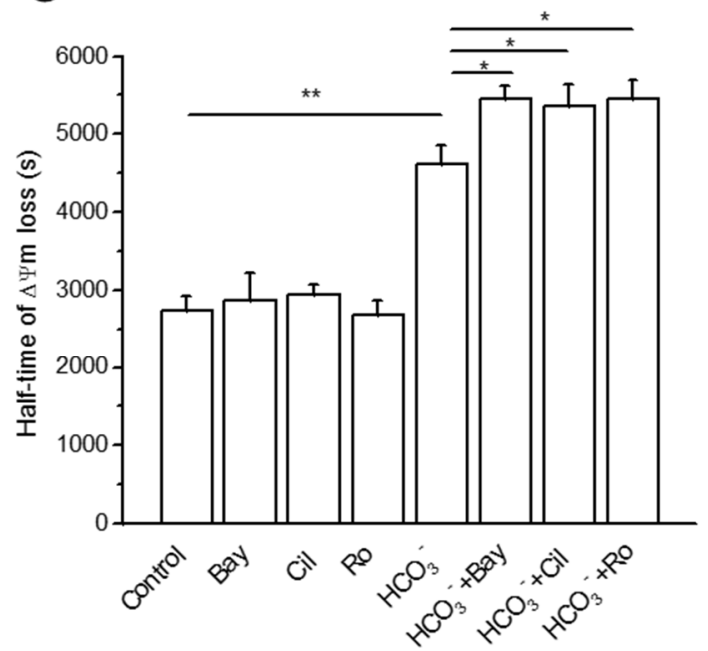

D

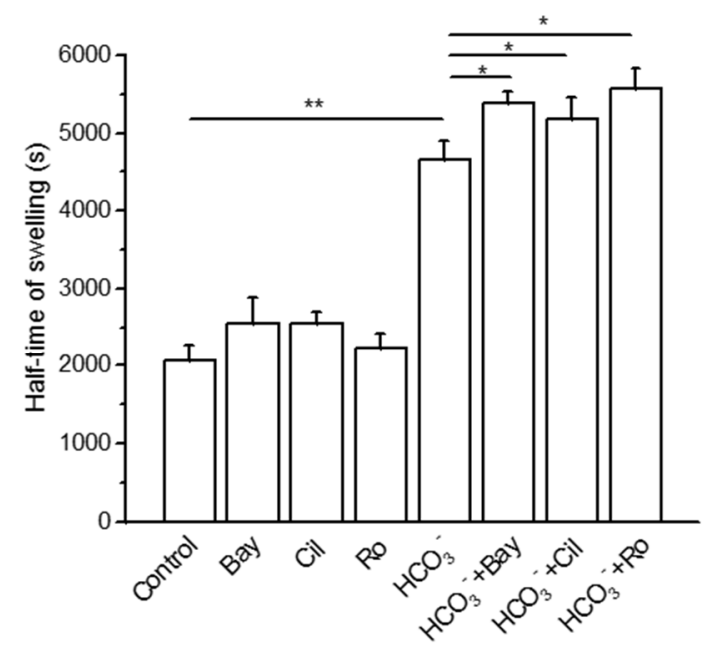

Fig. 4 


\section{Figure 4. PDEs regulate $\mathrm{Ca}^{2+}$-induced mitochondrial permeability transition (MPT).}

Evaluation of $\Delta \Psi \mathrm{m}$ loss (A) and mitochondrial swelling (B) induced by calcium in response to PDE2, PDE3 and PDE4 inhibition by the selective inhibitors Bay, Cil and Ro under basal condition or upon $\mathrm{HCO}_{3}{ }^{-}$stimulation. (C) Half-time of $\Delta \Psi \mathrm{m}$ loss induced by $10 \mu \mathrm{M} \mathrm{Ca}{ }^{2+}$ calculated from A. (D) Half-time of swelling induced by $10 \mu \mathrm{M} \mathrm{Ca}^{2+}$ calculated from B. PDE inhibitors were used at the same concentrations as in Fig. 3. Statistical analysis was performed using one way ANOVA. $* P<0.05, * * P<0.01$.

\subsection{Cardiospecific PDE2 transgenic overexpression influences mitochondrial function}

To examine more deeply the role of PDE2 in mitochondria, we took advantage of the heart specific PDE2A3 transgenic mouse line we generated and characterized previously ${ }^{30}$. First, we checked the expression of PDE2 specifically in mitochondria isolated from wild type (WT) and transgenic (TG) mice by subcellular fractionation and western-blot (Fig. 5A and B). The PDE2A level was about 20 times higher in SSM from PDE2 TG mice than in WT mice. Moreover, we observed by immunolabelling and confocal microscopy that overexpression of PDE2 in mice leads to a major expression of the protein at the plasma membrane of cardiomyocytes (Fig 5C). Since the labelling of mitochondria by MitoTracker Red shows a distribution of the organelles within the whole cell, a fraction of overexpressed PDE2 could in situ colocalize with SSM near the plasma membrane (Fig. 5C). In addition, the Pearson's correlation coefficient between PDE2 green fluorescence and red MitoTracker fluorescence, which is 0.418 , is in favor of a partial colocalization of PDE2 and mitochondria (Fig. 5C). Altogether, the results of western-blot and immunostaining allow us to propose that in transgenic PDE2A3 mice, the protein is mainly located at the plasma membrane and that a fraction of the protein has a subsarcolemmal mitochondrial localization. 
Then, we measured the mitochondrial $\mathrm{Ca}^{2+}$ accumulation with Rhod 2 in SSM mitochondria from WT and TG mice. Interestingly, we observed that mitochondrial $\mathrm{Ca}^{2+}$ accumulation is faster in isolated mitochondria from TG than in WT mice (Fig. 5D) and modulated by $\mathrm{HCO}_{3}{ }^{-}$ and the soluble $\mathrm{AC}$ inhibitor, 2 hydroxyestradiol $(2 \mathrm{HE}) . \mathrm{HCO}_{3}{ }^{-}$was less efficient in TG mice and WT to stimulate $\mathrm{Ca}^{2+}$ accumulation (Fig. 5E). cGMP had no effect on $\mathrm{Ca}^{2+}$ accumulation (not shown). 
A

Overexpression of PDE2 in subsarcolemmal

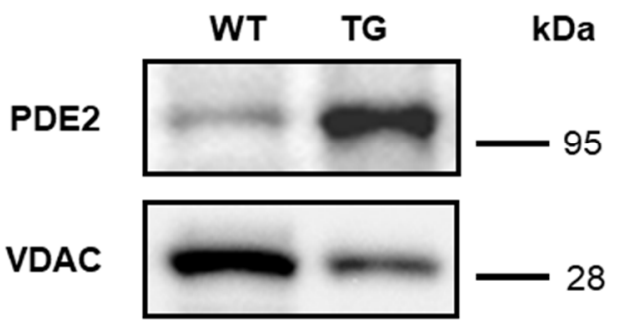

mitochondria impacts on

accumulation.

(A)

C

level of

in wild

(WT) and

transgenic

Alpha actinin

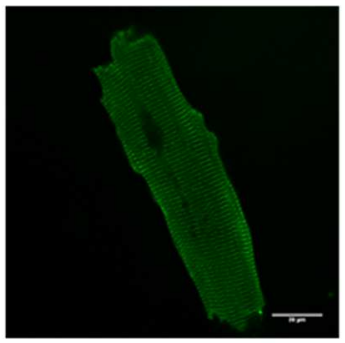

(TG)

was

western-

protein per

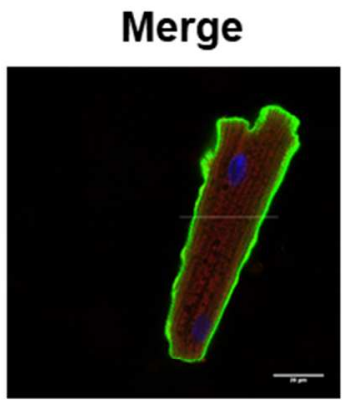

and quantified (B). (C)

PDE2 was
cardiomyocytes
mitochondrial network
actinin were revealed
labelling and

( 3000

든 2800

2400

2200

2000

1800

气̂ 1600

응 1400

¿ 1200

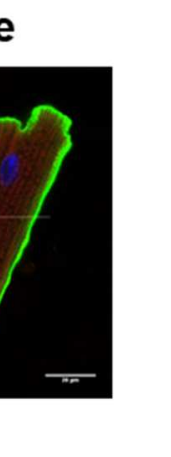

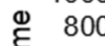

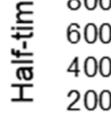

Mitotracker
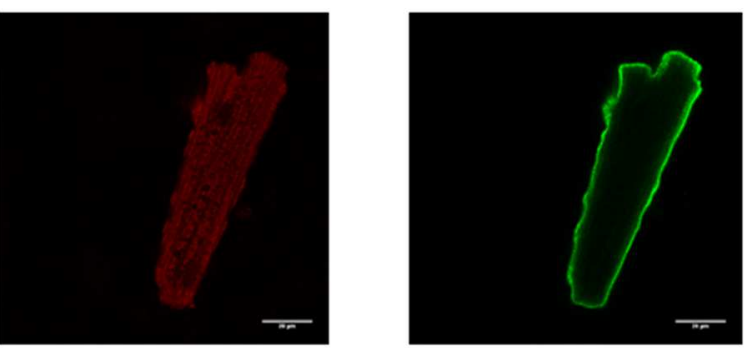

Expression

PDE2

type

mice

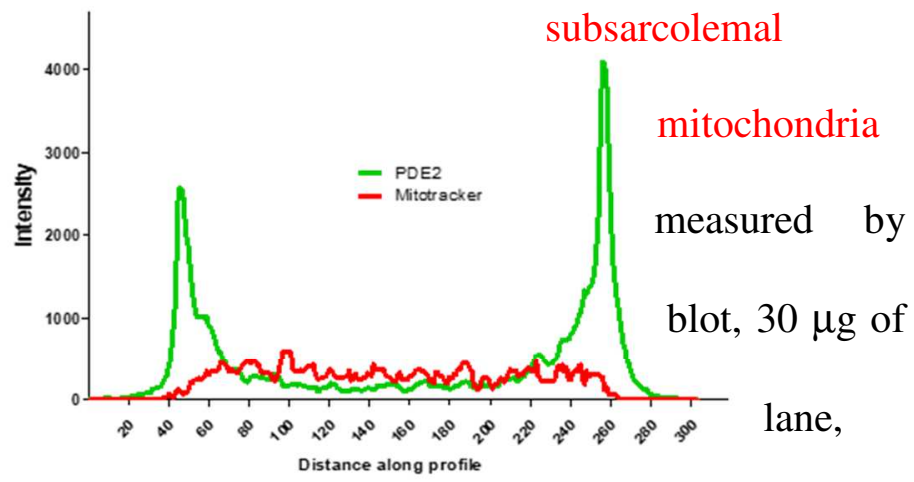

$\square$ WT $\square$ TG

Subcellular location of

evaluated by immunostaining of isolated from TG mice. The and the fibrillar protein alpha

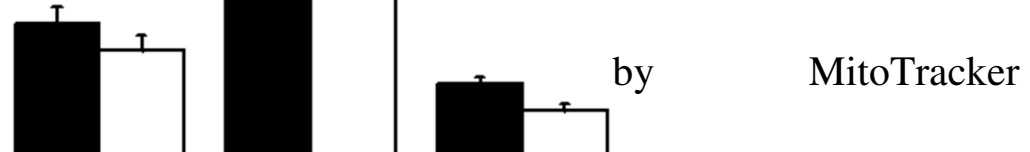
immunostaining,

Fig. 5 
respectively. Fluorescence intensity profiles of PDE2 green, MitoTracker red traced along the line. (D) Mitochondrial calcium accumulation was delayed by PDE2 overexpression in conditions of cAMP production stimulation by $15 \mathrm{mM} \mathrm{HCO}_{3}^{-}$, but not in control conditions or conditions of inhibition of cAMP production by $25 \mu \mathrm{M} 2 \mathrm{HE}$. Experiments were repeated three times. (B) shows means \pm s.e.m. using one way ANOVA. $* * P<0.01$. (D) shows means \pm s.e.m using two way ANOVA, Sidak's multiple comparisons. $* * P<0.01$.

Accordingly, the overexpression of PDE2 also influenced MPT induction, since SSM isolated from PDE2 TG mice showed a faster $\mathrm{Ca}^{2+}{ }_{\text {-induced }} \Delta \Psi \mathrm{m}$ loss and swelling compared to mitochondria isolated from WT mice (Fig. 6A-D). This was observed in basal condition as well as in conditions of sAC stimulation by $\mathrm{HCO}^{-}$and inhibition by $2 \mathrm{HE}$ (Fig. 6A-D). Of note, we observed no modulation of bicarbonate effect by $100 \mathrm{nM}$ Bay 60-7550 in TG mitochondria (not shown). This dose is the classical dose used that doesn't elicit unspecific effects on other PDEs, and thus, our results reveal the limitation of the PDE2 TG model for pharmacological study of PDE2 function in mitochondria. 

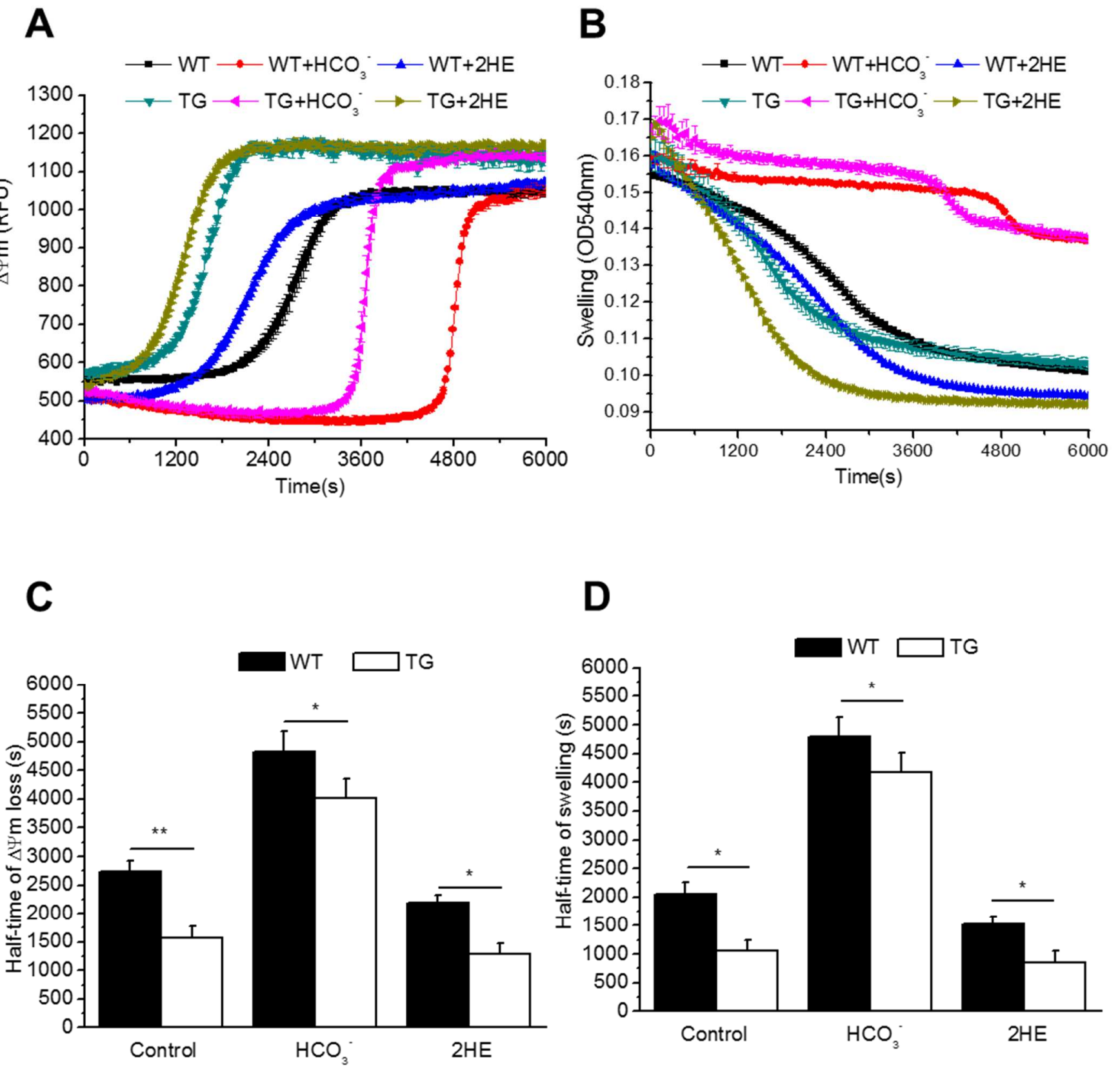

Fig. 6 


\section{Figure 6. PDE2 regulates $\mathrm{Ca}^{2+}$-induced mitochondrial permeability transition (MPT).}

Evaluation of $\Delta \Psi \mathrm{m}$ loss (A) or swelling (B) induced by calcium in cardiac mitochondria isolated from WT and PDE2 transgenic mice treated or not by $15 \mathrm{mM} \mathrm{HCO}_{3}{ }^{-}$and $15 \mu \mathrm{M}$ 2HE. Experiments were repeated three times. The bar graphs in (C) and (D) indicate the mean \pm s.e.m. and statistical analysis was performed using two way ANOVA, Sidak's multiple comparisons. $* P<0.05$, $* * P<0.01$.

Finally, we examined the bioenergetic function of mitochondria from WT and TG mice. We assessed mitochondrial respiration using the Seahorse XFe 96 machine, which allows real-time assessment of oxygen consumption rates (OCR) in isolated mitochondria. Thus, succinate was used as a substrate to feed electrons to complex II and basal respiration was measured. State 3 respiration was determined in the presence of ADP. Oligomycin (Oligo) addition blocked ATP synthase and so evoked state 40 respiration. Maximal respiration (state $3 \mathrm{u}$ ) was measured in the presence of the uncoupler FCCP. Finally, antimycin A (AA) was added to inhibit complex III. Compared to mitochondria isolated from WT mice, those from PDE2 TG mice exhibited a lower rate in complex II-driven basal respiration, in ADP-driven state 3 respiration and in FCCP-induced state $3 \mathrm{u}$ respiration (Fig. 7A and B). Moreover, we studied the effect of $5 \mu \mathrm{M}$ cGMP stimulation as a pretreatment of mitochondria (see material and methods). We observed no difference in OCR in basal conditions in WT and TG in the presence of cGMP. Similarly, we observed no effect of cGMP on state 3 and $3 \mathrm{u}$ respiration in WT mitochondria but in contrast, cGMP stimulated the state 3 and $3 \mathrm{u}$ respiration in TG mitochondria. 
This led us to propose a scheme indicating that mitochondrial cAMP level can be increased by sAC and decreased by PDE2 activity. This cAMP acts as a molecular switch controlling mitochondrial calcium entry, and then, favoring OXPHOS and/or MPT sensitivity (Fig. 7C).

A

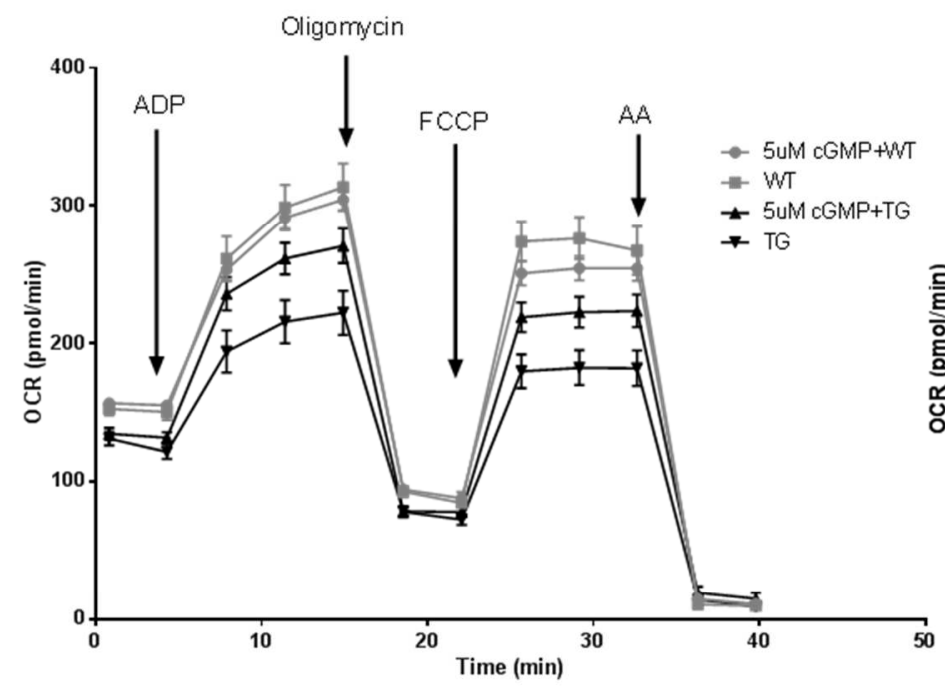

B

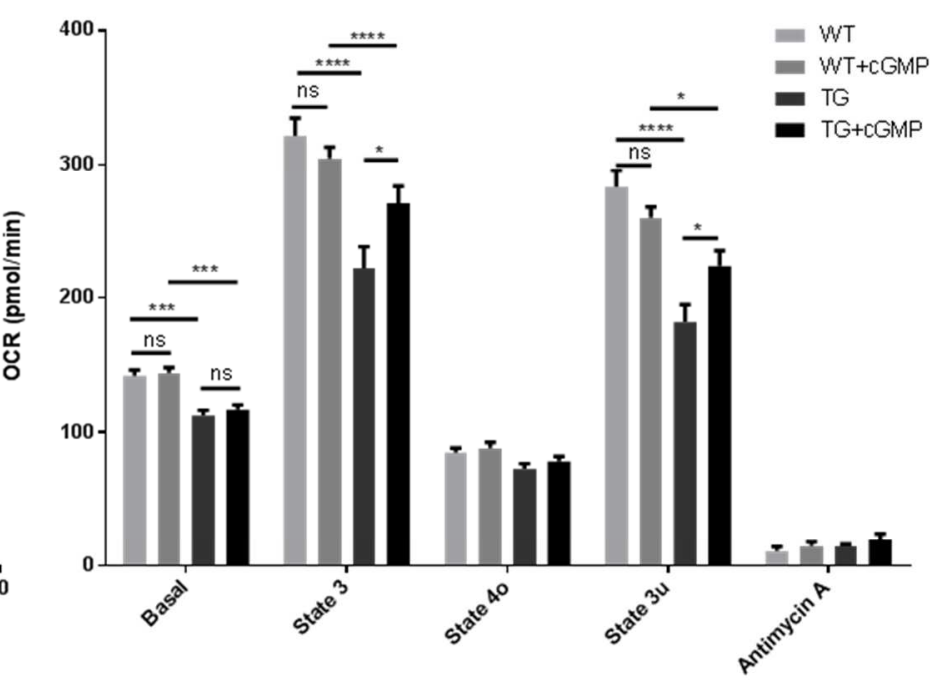

C

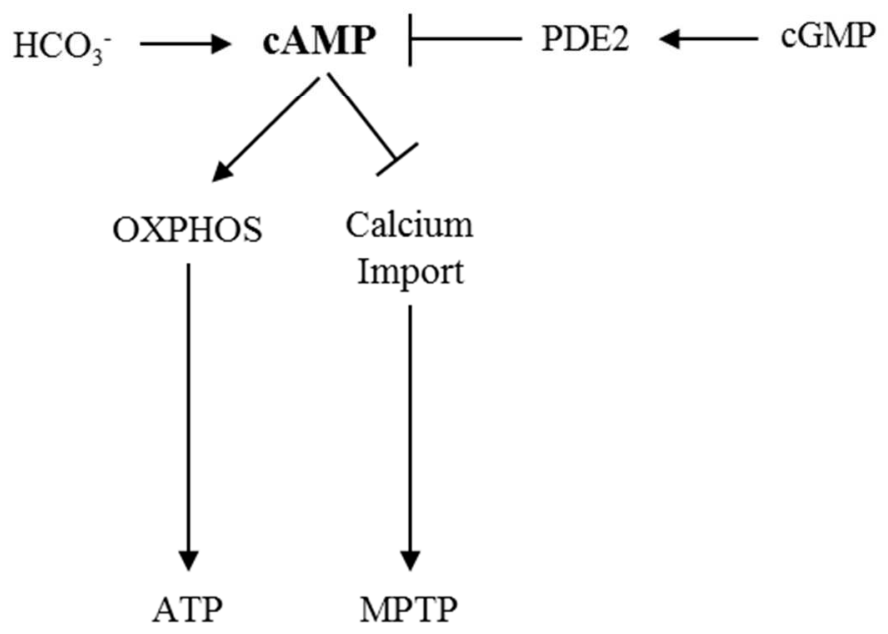

Fig. 7 
Figure 7. Bioenergetic analysis in isolated heart mitochondria from WT and PDE2 TG mice. (A) Representative graph of oxygen consumption rate (OCR) data are shown with succinate as the substrate followed by addition of ADP, oligomycin, FCCP, and antimycin A. (B) Average OCR profile calculated from panel (A). The results in bar graphs were analyzed by two way ANOVA, Sidak's multiple comparisons. Statistical significance is indicated as following: mean \pm s.e.m, $* P<0.05$, $* * * P<0.001$, $* * * * P<0.0001$ versus $\mathrm{WT}(\mathrm{n}=3)$. (C) Model for the regulation of cAMP pool by PDE2, cGMP and sAC: high levels of mitochondrial cAMP leads to OXPHOS stimulation, ATP production and MPT inhibition, whereas low levels of cAMP decreases OXPHOS and sensitize mitochondria to $\mathrm{Ca}^{2+}{ }_{\text {-induced MPT. }}$

\section{Discussion}

Our results show the expression of various PDEs in cardiac mitochondria and demonstrate their role in the control of cAMP level inside mitochondria, which regulates mitochondrial functions such as $\Delta \Psi \mathrm{m}$, respiration and $\mathrm{Ca}^{2+}$-dependent MPT. Among the 3 PDEs identified by western-blot (i.e. PDE2, 3 and 4), PDE2 was demonstrated to locate predominantly in the SSM in association with the inner membrane by electron microscopy and to limit mitochondrial level of cAMP.

Cyclic nucleotide signalling pathways regulate a vast number of physiological processes, including cell proliferation and differentiation, gene expression, apoptosis and metabolic pathways such as steroidogenesis, insulin secretion and glycogen synthesis $11,31,32$. Once synthesized by adenylyl cyclases or guanylyl cyclases, respectively, cAMP and cGMP transduce signal-encoded information by acting through a number of cellular effectors. Then, cAMP and cGMP signals are hydrolyzed by PDEs, allowing to restore cell homeostasis ${ }^{11,31}$, 32. 
Recently, the existence of a mitochondrial cAMP signalling pathway has been evidenced in various organs such as brain, liver and heart. It was also shown that cAMP increases ATP production in cells and isolated mitochondria ${ }^{4,33}$. A decrease in mitochondrial cAMP level by sAC inhibition decreases ATP production, $\mathrm{O}_{2}$ consumption, and cytochrome oxidase (COX) activity, whereas sAC activation by $\mathrm{HCO}_{3}{ }^{-}$and $\mathrm{Ca}^{2+}$ stimulates oxidative phosphorylation ${ }^{33}$, ${ }^{4}$. Different PDE families and subtypes were found in various subcellular compartments including nucleus, endoplasmic reticulum, sarcoplasmic reticulum and Golgi ${ }^{34,35}$. However, little was known about mitochondrial PDEs. Until now, only PDE4 and PDE2 have been found in mitochondria ${ }^{5,15,16}$. Acin-Perez et al. found that mitochondrial PDE2A is located in the matrix and that the unique $\mathrm{N}$ terminus of PDE2A isoform 2 specifically leads to mitochondrial localization of this isoform in rodent liver and brain ${ }^{5}$. PDE4B was reported to partially colocalize with DISC1 in the mitochondria of SH-SY5Y and LAN5 cells ${ }^{15}$. Asirvatham et al. showed that mitochondrial AKAP149 binds to PKA and PDE4A comprising regulatory modules to maintain cAMP homeostasis in the $\mathrm{T}$ cell ${ }^{36}$. In this study, we found PDE2A, 3A, 4A, 4B and 4D expression in mitochondrial matrix from rat heart isolated by western blot and precised the PDE2 inner membrane anchorage by transmission electron microscopy. In addition, we detected heterologous PDE2A3 and endogenous PDE2 isoforms in SSM by immunolabelling and confocal microscopy, despite the absence of in silico predictable mitochondrial targeting sequence. Indeed, MitoFates software was unable to predict a cleavable mitochondrial presequence in PDE2A3, but identified the short sequence LQRAV, which is a TOM20 interaction domain, TOM20 being a component of the TOM (translocase of outer membrane) receptor complex responsible for the recognition and translocation of cytosolically synthesized mitochondrial preproteins (not shown), raising the possibility of a mitochondrial localization of PDE2A3. 
Acin-Perez et al. showed that PDE2A was present in brain and liver mitochondrial fractions and was responsible for cAMP-hydrolysis. In contrast, PDE4 had no significant contribution to cAMP-hydrolysis in liver mitochondria, but showed almost the same activity as PDE2 in brain mitochondria ${ }^{5}$. In isolated rat heart mitochondria, we showed that PDE2 represented the largest mitochondrial cAMP-degrading PDE activity, followed by PDE3 and PDE4 and that PDE2 can be activated by cGMP (see table 1). Our cardiac mitochondrial PDE2 activity correlated with Acin-Perez's data, thus suggesting that PDE2 is the main PDE in heart, liver and brain mitochondria. However, in neonatal cardiomyocytes infected with $4 \mathrm{mt}-\mathrm{Epac}-\mathrm{S}^{\mathrm{H} 187}$, inhibition of PDE2 and 3 caused about $2.5 \%$ increase in FRET signal whereas inhibition of PDE4 resulted in $25 \%$ increase in basal condition. Upon $\mathrm{HCO}_{3}^{-}$stimulation, i.e. upon increased cAMP levels, inhibition of PDE2 and 3 caused $40 \%$ increase in cAMP, whereas it reached up to $80 \%$ with PDE4 inhibition. This might be due to a difference in PDE2 activity in isolated mitochondria from adult rat heart versus neonatal cardiomyocytes, which is not observed for PDE3 and PDE4 and remains to be confirmed.

In cardiac mitochondria, we have shown that mitochondrial cAMP stimulates $\mathrm{O}_{2}$ consumption and ATP production ${ }^{8}$. Consistent with this, Acin-Perez et al. reported that inhibition of PDE2 caused an increase in $\mathrm{O}_{2}$ consumption and ATP production in mouse brain mitochondria. Here, using PDE2 TG mice, we showed that SSM isolated from PDE2 TG mice had a lower $\mathrm{O}_{2}$ consumption rate than WT mice, but higher when mitochondria are stimulated by cGMP. This suggest a complex metabolic regulation of mitochondrial energetic metabolism by PDEs depending on the ratios of PDE2 and 3 isoforms and the level of cGMP by an unknown mechanism. The investigation of this mechanism might provide new insights on the regulation of cardiac cell function and dysfunction, which are energy-demanding (i.e. contraction, arrhythmias)._In addition, we previously reported that cAMP produced within mitochondria regulates $\mathrm{Ca}^{2+}$ accumulation and MPT ${ }^{8}$. Here, we found that upon $\mathrm{HCO}_{3}{ }^{-}$ 
stimulation to increase cAMP, inhibition of PDE2, PDE3 and PDE4 delayed these processes, although inhibition of PDEs did not have an effect in basal condition. Moreover, SSM isolated from PDE2 TG mice showed a faster $\Delta \Psi \mathrm{m}$ loss and swelling compared to mitochondria isolated from WT mice confirming the results obtained in isolated mitochondria treated with PDE2 inhibitor. Since increased PDE2A expression was reported in human heart failure ${ }^{21}$, PDE2A inhibition could be seen as a therapeutic strategy to improve heart metabolism and protect from apoptotic cell death, as recently suggested ${ }^{17}$. However, no adverse effects were observed so far in heart-specific PDE2 TG mice ${ }^{30}$. On the contrary, PDE2 TG mice are protected against arrhythmias and show an improved contraction force after severe ischemic insult ${ }^{30}$. Thus, further work is needed to explore the pathophysiological roles of PDE2 in models of heart disease as well as to look more closely at its action on distinct cellular cAMP and cGMP pools ${ }^{37}$.

In conclusion, our present work demonstrates that cAMP degrading PDEs form a mitochondrial signalling pathway controlling cAMP produced locally by sAC, and regulate mitochondrial $\mathrm{Ca}^{2+}$ accumulation and MPT. PDE2, 3 and 4 are localized in cardiac SSM and contribute to the hydrolysis of mitochondrial cAMP, regulate $\mathrm{Ca}^{2+}$ accumulation and MPT and, eventually, cell survival and MPT-mediated cell death. Given the role of ATP and $\mathrm{Ca}^{2+}$ in the heart, our findings may have implications for the metabolic control of cardiac function.

\section{Conflict of Interest}

The authors declare no conflict of interest.

\section{Acknowledgments}

This work has been funded by INSERM (CB, DM, GV, RF), the Investment for the Future program ANR-11-IDEX-0003-01 within the LABEX ANR-10-LABX-0033 (CB, DM, GV, 
RF), ANR-13-ISV1-0001-01 (CB), ANR JCJC ANR-16-CE14-0014 grant (DM) and

ANR13BSV10003-02 (GV). We thank C. Longin for TEM microscopy (platform MIMA2,

INRA Jouy en Josas, France) and Dr B. Manoury for his expertise in statistical analysis.

Zhenyu WANG and Dawei LIU were supported by a fellowship from the China Scholarship

Council. The Seahorse apparatus was co-funded by CORDDIM (Investissement, 2015) and

IPSIT, Châtenay-Malabry, France.

\section{References}

1. Brenner C, Kroemer G. Apoptosis. Mitochondria--the death signal integrators. Science 2000, 289(5482): 1150-1151.

2. Weinberg SE, Chandel NS. Targeting mitochondria metabolism for cancer therapy. Nature chemical biology 2015, 11(1): 9-15.

3. Vandecasteele G, Szabadkai G, Rizzuto R. Mitochondrial calcium homeostasis: mechanisms and molecules. IUBMB life 2001, 52(3-5): 213-219.

4. Acin-Perez R, Salazar E, Kamenetsky M, Buck J, Levin LR, Manfredi G. Cyclic AMP produced inside mitochondria regulates oxidative phosphorylation. Cell metabolism 2009, 9(3): 265-276.

5. Acin-Perez R, Russwurm M, Gunnewig K, Gertz M, Zoidl G, Ramos L, et al. A phosphodiesterase $2 \mathrm{~A}$ isoform localized to mitochondria regulates respiration. The Journal of biological chemistry 2011, 286(35): 30423-30432.

6. Appukuttan A, Kasseckert SA, Micoogullari M, Flacke J-P, Kumar S, Woste A, et al. Type 10 adenylyl cyclase mediates mitochondrial Bax translocation and apoptosis of adult rat cardiomyocytes under simulated ischaemia/reperfusion. Cardiovascular research 2011, 93(2): 340-349.

7. Kumar S, Kostin S, Flacke J-P, Reusch HP, Ladilov Y. Soluble adenylyl cyclase controls mitochondria-dependent apoptosis in coronary endothelial cells. Journal of Biological Chemistry 2009, 284(22): 14760-14768.

8. Wang Z, Liu D, Varin A, Nicolas V, Courilleau D, Mateo P, et al. A cardiac mitochondrial cAMP signaling pathway regulates calcium accumulation, permeability transition and cell death. Cell death \& disease 2016, 7: e2198.

9. Bender AT, Beavo JA. Cyclic nucleotide phosphodiesterases: molecular regulation to clinical use. Pharmacological reviews 2006, 58(3): 488-520. 
10. Sharma S, Kumar K, Deshmukh R, Sharma PL. Phosphodiesterases: Regulators of cyclic nucleotide signals and novel molecular target for movement disorders. European journal of pharmacology 2013, 714(1-3): 486-497.

11. Conti M, Beavo J. Biochemistry and physiology of cyclic nucleotide Phosphocliesterases: Essential components in cyclic nucleotide signaling. Annual review of biochemistry 2007, 76: 481-511.

12. Omori K, Kotera J. Overview of PDEs and their regulation. Circulation research 2007, 100(3): 309-327.

13. Middelhaufe S, Garzia L, Ohndorf UM, Kachholz B, Zollo M, Steegborn C. Domain mapping on the human metastasis regulator protein h-Prune reveals a C-terminal dimerization domain. The Biochemical journal 2007, 407(2): 199-205.

14. D'Angelo A, Garzia L, Andre A, Carotenuto P, Aglio V, Guardiola O, et al. Prune cAMP phosphodiesterase binds $\mathrm{nm} 23-\mathrm{H} 1$ and promotes cancer metastasis. Cancer cell 2004, 5(2): 137-149.

15. Millar JK, Pickard BS, Mackie S, James R, Christie S, Buchanan SR, et al. DISC1 and PDE4B are interacting genetic factors in schizophrenia that regulate cAMP signaling. Science 2005, 310(5751): 1187-1191.

16. Modis K, Panopoulos P, Coletta C, Papapetropoulos A, Szabo C. Hydrogen sulfidemediated stimulation of mitochondrial electron transport involves inhibition of the mitochondrial phosphodiesterase 2A, elevation of cAMP and activation of protein kinase A. Biochem Pharmacol 2013, 86(9): 1311-1319.

17. Monterisi S, Lobo MJ, Livie C, Castle JC, Weinberger M, Baillie G, et al. PDE2A2 regulates mitochondria morphology and apoptotic cell death via local modulation of cAMP/PKA signalling. Elife 2017, 6: e21374.

18. Zhang F, Qi Y, Zhou K, Zhang G, Linask K, Xu H. The cAMP phosphodiesterase Prune localizes to the mitochondrial matrix and promotes mtDNA replication by stabilizing TFAM. EMBO reports 2015: e201439636.

19. Kilkenny C, Browne W, Cuthill IC, Emerson M, Altman DG. Animal research: reporting in vivo experiments: the ARRIVE guidelines. Brit J Pharmacol 2010, 160(7): $1577-1579$.

20. Thompson WJ, Appleman MM. Characterization of cyclic nucleotide phosphodiesterases of rat tissues. The Journal of biological chemistry 1971, 246(10): 3145-3150.

21. Mehel H, Emons J, Vettel C, Wittkopper K, Seppelt D, Dewenter M, et al. Phosphodiesterase-2 Is Up-Regulated in Human Failing Hearts and Blunts betaAdrenergic Responses in Cardiomyocytes. J Am Coll Cardiol 2013, 62(17): 15961606. 
22. Coletta C, Modis K, Olah G, Brunyanszki A, Herzig DS, Sherwood ER, et al. Endothelial dysfunction is a potential contributor to multiple organ failure and mortality in aged mice subjected to septic shock: preclinical studies in a murine model of cecal ligation and puncture. Crit Care 2014, 18(5).

23. Morel E, Marcantoni A, Gastineau M, Birkedal R, Rochais F, Garnier A, et al. cAMPbinding protein Epac induces cardiomyocyte hypertrophy. Circulation research 2005, 97(12): 1296-1304.

24. Klarenbeek J, Goedhart J, van Batenburg A, Groenewald D, Jalink K. Fourthgeneration epac-based FRET sensors for cAMP feature exceptional brightness, photostability and dynamic range: characterization of dedicated sensors for FLIM, for ratiometry and with high affinity. PloS one 2015, 10(4): e0122513.

25. Bolte $\mathrm{S}$, Cordelieres F. A guided tour into subcellular colocalization analysis in light microscopy. Journal of microscopy 2006, 224(3): 213-232.

26. Abi-Gerges A, Richter W, Lefebvre F, Matéo P, Varin A, Heymes C, et al. Decreased expression and activity of cAMP phosphodiesterases in cardiac hypertrophy and its impact on B-adrenergic cAMP signals. Circulation research 2009, 105: 784-792.

27. Martins TJ, Mumby M, Beavo J. Purification and characterization of a cyclic GMPstimulated cyclic nucleotide phosphodiesterase from bovine tissues. Journal of Biological Chemistry 1982, 257(4): 1973-1979.

28. Zoratti M, Szabò I. The mitochondrial permeability transition. Biochimica et Biophysica Acta (BBA)-Reviews on Biomembranes 1995, 1241(2): 139-176.

29. Belzacq-Casagrande A-S, Martel C, Pertuiset C, Borgne-Sanchez A, Jacotot E, Brenner C. Pharmacological screening and enzymatic assays for apoptosis. Front Biosci 2009, 14: 3550-3562.

30. Vettel C, Lindner M, Dewenter M, Lorenz K, Schanbacher C, Riedel M, et al. Phosphodiesterase 2 protects against catecholamine-induced arrhythmia and preserves contractile function after myocardial infarction. Circulation research 2016, 62: 15961606

31. Francis SH, Blount MA, Corbin JD. Mammalian Cyclic Nucleotide Phosphodiesterases: Molecular Mechanisms and Physiological Functions. Physiol Rev 2011, 91(2): 651-690.

32. Keravis T, Lugnier C. Cyclic nucleotide phosphodiesterase (PDE) isozymes as targets of the intracellular signalling network: benefits of PDE inhibitors in various diseases and perspectives for future therapeutic developments. Brit J Pharmacol 2012, 165(5): 1288-1305.

33. Di Benedetto G, Scalzotto E, Mongillo M, Pozzan T. Mitochondrial Ca2+ uptake induces cyclic AMP generation in the matrix and modulates organelle ATP levels. Cell metabolism 2013, 17(6): 965-975. 
34. Lugnier C. Cyclic nucleotide phosphodiesterase (PDE) superfamily: a new target for the development of specific therapeutic agents. Pharmacology \& therapeutics 2006, 109(3): 366-398.

35. Maurice DH, Ke H, Ahmad F, Wang Y, Chung J, Manganiello VC. Advances in targeting cyclic nucleotide phosphodiesterases. Nature reviews Drug discovery 2014, 13(4): 290-314.

36. Asirvatham AL, Galligan SG, Schillace RV, Davey MP, Vasta V, Beavo JA, et al. Akinase anchoring proteins interact with phosphodiesterases in T lymphocyte cell lines. J Immunol 2004, 173(8): 4806-4814.

37. Wagner M, Mehel H, Fischmeister R, El-Armouche A. Phosphodiesterase 2: antiadrenergic friend or hypertrophic foe in heart disease? Naunyn-Schmiedeberg's archives of pharmacology 2016, 389(11): 1139-1141. 
